\title{
LA DISCIPLINA DELLA GIURISDIZIONE NEL REGOLAMENTO (UE) N. 2016/679 CONCERNENTE IL TRATTAMENTO DEI DATI PERSONALI E IL SUO COORDINAMENTO CON LA DISCIPLI- NA CONTENUTA NEL REGOLAMENTO "BRUXELLES I-BIS"
}

\author{
JURISDICTION UNDER REGULATION (EU) NO. 2016/679 CON- \\ CERNING THE PROCESSING OF PERSONAL DATA AND ITS \\ COORDINATION WITH THE “BRUSSELS I-BIS” REGULATION
}

\author{
Fabrizio Marongiu Buonaiuti \\ Professore ordinario di Diritto internazionale \\ Università di Macerata \\ orcid ID: 0000-0002-4188-8099
}

Recibido: 16.08.2017 / Aceptado: 11.09.2017

DOI: https://doi.org/10.20318/cdt.2017.3881

\begin{abstract}
Riassunto: Lo scritto esamina le regole di giurisdizione contenute nel regolamento (UE) $\mathrm{n}$. 2016/679 relativo alla protezione delle persone fisiche con riguardo al trattamento dei dati personali, le quali si presentano come strumentali all'obiettivo di garantire una tutela giurisdizionale effettiva dei diritti riconosciuti dal regolamento a favore degli individui titolari dei dati nei confronti dei soggetti responsabili del trattamento dei dati stessi. Tali regole, pur apprezzabili nel loro intento di fornire una disciplina specifica della giurisdizione relativamente alle controversie prese in considerazione, pongono nondimeno alcuni problemi di coordinamento con la disciplina della giurisdizione nelle controversie civili e commerciali come attualmente contenuta nel regolamento (UE) n. 1215/2012 o "Bruxelles I-bis", i quali non sono adeguatamente affrontati nelle disposizioni del nuovo trattamento.
\end{abstract}

Parole chiave: Giurisdizione, Regolamento (UE) n. 2016/679, trattamento dei dati personali, tutela giurisdizionale effettiva, Regolamento (UE) n. 1215/2012 ("Bruxelles I-bis").

Abstract: The present paper addresses the rules on jurisdiction contained in Regulation (EU) No. 2016/679 concerning the protection of natural persons with regard to the processing of personal data. These rules appear instrumental to the pursuit of the fundamental goal of guaranteeing an effective judicial protection of the rights granted to individuals as concerns the processing of their personal data against those subjects who are responsible for the said processing. These rules, welcome as they are in their purpose of providing special fora expressly tailored in respect of the peculiarities of the litigation concerned, fall short of addressing adequately the problems of coordination which they raise in respect of the rules on jurisdiction in civil and commercial matters as currently set out under Regulation (EU) No. 1215/2012 (s.c. "Brussels I $a$ ” Regulation).

Keywords: Jurisdiction; Regulation (EU) No. 2016/679, processing of personal data, right to an effective judicial remedy, Regulation (EU) No. 1215/2012 ("Brussels Ia").

Sommario: I. Obiettivi perseguiti dal nuovo regolamento sulla protezione delle persone fisiche relativamente al trattamento dei dati personali e collocazione sistematica delle regole sulla giurisdizione al suo interno. II. Le regole sulla giurisdizione relativamente alle azioni dei titolari dei dati personali nei confronti dei soggetti responsabili del trattamento, in rapporto alle regole sul foro 
del fatto illecito nel regolamento "Bruxelles I-bis": A. L'oggetto delle regole di giurisdizione contenute nel regolamento $\mathrm{n}$. 2016/679 e la possibile integrazione di tale disciplina con regole generali del regolamento "Bruxelles I-bis"; B. L'applicabilità delle regole di giurisdizione del regolamento n. 2016/679 nei confronti di titolari o responsabili del trattamento stabiliti in paesi terzi; C. L'opzione consentita dal regolamento a favore del foro della residenza abituale del titolare dei dati personali e le sue criticità, alla luce della parallela giurisprudenza della Corte di giustizia relativa alle violazioni della privacy e dei diritti della personalità; D. L'imperatività della tutela del titolare dei dati come giustificazione sistematica dell'opzione a favore del foro della sua residenza abituale. III. Le disposizioni concernenti la sospensione delle azioni in caso di procedimenti paralleli, in rapporto alla disciplina in materia di litispendenza e connessione c.d. privativa nel regolamento "Bruxelles I-bis". IV. Considerazioni conclusive: un'occasione mancata per un più efficace coordinamento tra atti adottati in settori diversi nell'ambito delle politiche materiali dell'Unione europea.

\section{Obiettivi perseguiti dal nuovo regolamento sulla protezione delle persone fisiche relativamente al trattamento dei dati personali e collocazione sistematica delle regole sulla giurisdizione al suo interno}

1. Il regolamento n. 2016/679 è destinato ad introdurre, a decorrere dal 25 maggio 2018, data a partire dalla quale riceverà applicazione, una disciplina uniforme in materia di protezione dei diritti delle persone fisiche relativamente al trattamento dei propri dati personali ${ }^{1}$, la quale sostituirà la disciplina attualmente in vigore, contenuta nella direttiva n. 95/46 e nelle discipline nazionali di recepimento. Lo scopo perseguito dal nuovo strumento consiste nell'offrire una più efficace garanzia dei diritti degli individui relativamente al trattamento dei propri dati personali, che la Carta dei diritti fondamentali dell'Unione europea espressamente annovera all'articolo 8 tra i diritti fondamentali della persona, rispetto a quella sinora apprestata a mezzo della direttiva n. 95/46, introducendo una disciplina maggiormente omogenea in materia, come è proprio della natura dell'atto che la racchiude, all'interno degli Stati membri. Nondimeno, pur nell'ambito di una disciplina tendenzialmente uniforme e direttamente applicabile, il regolamento intenzionalmente lascia un margine di discrezionalità agli Stati membri per quanto attiene alla disciplina del trattamento di speciali categorie di dati personali, costituite dai c.d. dati sensibili, nell'intento di contemperare l'obiettivo di introdurre una disciplina per quanto possibile omogenea in materia con la necessaria flessibilità per quanto attiene alla disciplina di ipotesi particolari².

2. Tra i principi ispiratori della disciplina contenuta nel regolamento rientra il diritto alla tutela giurisdizionale effettiva per il caso in cui i diritti tutelati dal regolamento stesso abbiano ad essere violati $^{3}$. Ai rimedi giurisdizionali o di carattere amministrativo a disposizione degli individui in relazione a violazioni dei diritti tutelati dal regolamento è dedicato il capitolo VIII del regolamento, il quale reca un meccanismo di tutela a due stadi, il primo del quale contempla, ai sensi dell'art. 77 del regolamento, il diritto di presentare un reclamo relativamente ad ogni violazione dei diritti tutelati dal regolamento innanzi ad un'autorità di controllo, da individuarsi alternativamente nell'autorità dello Stato membro di residenza abituale del titolare dei dati o in quella dello Stato membro dove egli svolge la propria attività lavorativa, ovvero nell'autorità dello Stato membro in cui la violazione è avvenuta. Tale rimedio viene indicato come alternativo al ricorso ad altri mezzi di carattere amministrativo o giurisdizionale a cui l'individuo possa avere accesso, tra i quali debbono ritenersi rientrare i rimedi giurisdizionali direttamente azionabili nei confronti del titolare del trattamento o del responsabile del trattamento ai sensi del suc-

\footnotetext{
1 Regolamento (UE) 2016/679 del Parlamento europeo e del Consiglio del 27 aprile 2016 relativo alla protezione delle persone fisiche con riguardo al trattamento dei dati personali, nonché alla libera circolazione di tali dati e che abroga la direttiva 95/46/CE (regolamento generale sulla protezione dei dati), in GUUE, L 119 del 4 maggio 2016, p. 1 ss.

2 Si vedano in questo senso le disposizioni degli articoli 9, par. 2, lett. a), g)-j) e parr. 3-4, art. 10 e art. 23 del regolamento, nonché i considerando nn. 10 e 73 del preambolo.

3 Si vedano gli articoli 78, par. 1, che afferma il diritto del titolare dei dati ad un rimedio giurisdizionale effettivo avverso decisioni sfavorevoli di un'autorità nazionale di controllo, e 79, par. 1, che afferma tale diritto nei confronti del titolare ovvero del responsabile del trattamento. Si veda anche il considerando n. 141 del preambolo, il quale contiene un espresso riferimento in proposito all'art. 47 della Carta dei diritti fondamentali dell'Unione europea.
} 
cessivo art. $79^{4}$. Il secondo stadio, susseguente alla presentazione di un reclamo ad un'autorità nazionale di controllo, contempla, ai sensi dell'art. 78 del regolamento, il diritto ad un rimedio giurisdizionale effettivo nei confronti di una decisione sfavorevole dell'autorità nazionale di controllo. In considerazione della natura di una tale autorità e della funzione di carattere pubblico che questa esercita, il regolamento necessariamente prevede che tali ricorsi debbano presentarsi innanzi ai giudici dello Stato membro nel quale l'autorità di controllo che ha emesso la decisione sfavorevole è istituita 5 .

3. Se, per quanto appena rilevato, particolari problemi non sono suscettibili di porsi in termini di giurisdizione per quanto attiene ai ricorsi nei confronti di decisioni sfavorevoli adottate da un'autorità nazionale di controllo ai sensi dell'art. 78 del regolamento, la problematica dell'allocazione della giurisdizione è invece concretamente suscettibile di porsi in relazione alla strada alternativa, contemplata dall'art. 79 del regolamento, dell'azione nei confronti del titolare o del responsabile del trattamento dei dati personali. Una tale azione, diversamente da quella nei confronti di una decisione sfavorevole dell'autorità di controllo, presenterà il carattere di un'ordinaria azione di carattere civile, la quale potrà essere volta, a seconda dei casi, ad ottenere la cessazione o la modificazione della condotta del titolare ovvero del responsabile del trattamento che non sia conforme alle disposizioni del regolamento, oppure, ed eventualmente anche parallelamente, al risarcimento del danno causato dalla violazione, come espressamente contemplato dall'art. 82 del regolamento. Conseguentemente, nei casi in cui il titolare dei dati personali e il titolare ovvero il responsabile del trattamento abbiano rispettivamente la propria residenza abituale e il proprio luogo di stabilimento in paesi membri diversi, ovvero la violazione sia avvenuta in un diverso paese membro - situazioni le quali sono rese innegabilmente più frequenti dal sempre più diffuso ricorso a mezzi di carattere telematico per la conclusione del più vario genere di transazioni, le quali implicano l'acquisizione e il trattamento di dati personali degli utenti - sono frequentemente suscettibili di porsi i problemi di determinazione della giurisdizione che sono tipici delle controversie civili di carattere internazionale, questi formano oggetto di una rete ormai sempre più fitta di strumenti adottati dall'Unione europea nel settore della cooperazione giudiziaria in materia civile. Particolare rilievo occupa in questo ambito il regolamento (UE) n. 1215/2012 o "Bruxelles I-bis", relativo alla disciplina della competenza giurisdizionale nonché del riconoscimento e dell'esecuzioni delle decisioni in materia civile e commerciale'.

\section{Le regole sulla giurisdizione relativamente alle azioni dei titolari dei dati personali nei con- fronti dei soggetti responsabili del trattamento, in rapporto alle regole sul foro del fatto illecito nel regolamento "Bruxelles I-bis".}

\section{(A) L'oggetto delle regole di giurisdizione contenute nel regolamento $\mathrm{n}$. 2016/679 e la possibile integrazione di tale disciplina con regole generali del regolamento "Bruxelles I-bis".}

4. Il regolamento n. 2016/679 disciplina, al par. 2 dell'art. 79, la giurisdizione relativamente alle azioni del titolare dei dati personali nei confronti del titolare o del responsabile del trattamento, adottan-

\footnotetext{
${ }^{4}$ Per chiarezza, anche a beneficio della successiva indagine, si deve precisare che il regolamento contempla due distinte figure indicate rispettivamente come "titolare del trattamento" e come "responsabile del trattamento". Relativamente al primo, l'art. 4, n. 7, del regolamento chiarisce che come tale si deve intendere la persona fisica o giuridica, il servizio o organismo od anche, a seconda dei casi, l'autorità pubblica, che individua i mezzi e le finalità del trattamento in conformità delle disposizioni del regolamento e, ove del caso, di altre disposizioni del diritto dell'Unione europea ovvero del diritto interno dei singoli Stati membri, mentre per il secondo deve intendersi, ai sensi dell'art. 4, n. 8, chi materialmente effettua il trattamento per conto del titolare del trattamento.

5 Si veda l'art. 78, par. 3. Analogamente, come si vedrà meglio infra, II.4, l'art. 79, par. 2, del regolamento prevede che le azioni nei confronti del titolare ovvero del responsabile del trattamento possano introdursi unicamente innanzi ai giudici dello Stato membro in cui il titolare o il responsabile del trattamento abbia uno stabilimento, nell'ipotesi in cui il titolare ovvero il responsabile del trattamento sia una pubblica autorità che agisca nell'esercizio dei propri pubblici poteri.

${ }^{6}$ Relativamente ai rapporti con quest'ultimo regolamento, come si avrà modo di osservare più avanti, II.4, il regolamento n. 2016/679 si limita ad affermare, nel considerando n. 147 del preambolo, che in presenza di specifiche regole in materia di giurisdizione contenute nel regolamento stesso, le regole generali di giurisdizione come contenute, segnatamente, nel regolamento n. 1215/2012 o "Bruxelles I-bis", non dovranno pregiudicare l'applicazione di tali regole specifiche.
} 
do soluzioni che, pur in assenza di un riferimento espresso a tale regolamento nel testo delle norme in esame, appaiono largamente ispirate ai criteri propri del regolamento n. 1215/2012 o "Bruxelles I-bis" e all'interpretazione che di tali criteri, come già in larga parte contenuti nel previgente regolamento $\mathrm{n}$. 44/2001 o "Bruxelles I" ed ancor prima nell'originaria Convenzione di Bruxelles del 1968, è stata data nel tempo dalla Corte di giustizia. Peraltro, secondo un'indicazione di carattere generale contenuta nel considerando n. 147 del preambolo, si deve ritenere che i criteri recati dalla norma in esame siano destinati a prevalere, in virtù di un criterio di specialità ratione materiae accolto dal regolamento Bruxelles Ibis nel suo art. 67, sulle regole generali contenute in quest'ultimo regolamento, le quali potranno trovare applicazione solo nella misura in cui non siano incompatibili con la disciplina speciale ${ }^{7}$.

5. Deve a questo proposito osservarsi che i criteri speciali contemplati dall'art. 79, par. 2 del regolamento concernente la tutela dei dati personali, conformemente all'ottica protettiva della persona del titolare dei dati che, come si è rilevato, ispira l'intera disciplina recata dal regolamento e coerentemente col par. 1 della stessa norma, che tutela il diritto del titolare dei dati ad un rimedio giurisdizionale effettivo, si applicano unicamente alle azioni promosse dal titolare dei dati nei confronti del titolare o del responsabile del trattamento dei dati stessi ${ }^{8}$. Per quanto concerne le eventuali azioni di accertamento negativo che il titolare ovvero il responsabile del trattamento intendessero proporre nei confronti del titolare dei dati, come pure le eventuali azioni promosse dal titolare del trattamento nei confronti del responsabile del trattamento stesso o viceversa, deve ritenersi che, secondo lo spirito del considerando n. 147 del regolamento, che, come già si è osservato, replica la regola di coordinamento dettata dall'art. 67 del regolamento "Bruxelles I-bis", esse rimangano disciplinate in punto di giurisdizione da quest'ultimo regolamento, le cui disposizioni, come si avrà modo di osservare, disciplineranno anche eventuali situazioni di concorso tra procedimenti instaurati dai titolari dei dati in base al primo regolamento ed eventuali procedimenti introdotti dal titolare ovvero dal responsabile del trattamento in base al secondo?

6. Sempre secondo la medesima ratio di complementarità della disciplina recata dal regolamento "Bruxelles I-bis" rispetto alle regole in materia di giurisdizione contenute nel regolamento n. 679/2016, deve ritenersi che le disposizioni sulla competenza per connessione contenute nel primo regolamento, con particolare riferimento alle ipotesi di chiamate in garanzia e di domande riconvenzionali proposte dal titolare o dal responsabile del trattamento, come anche, ove del caso, la disposizione relativa al cumulo soggettivo di cui all'art. 8, par. 1 del regolamento Bruxelles I-bis - la cui applicazione può giovare ad una più effettiva tutela giurisdizionale dei diritti del titolare dei dati, nel caso, ad esempio, in cui egli intenda agire al tempo stesso nei confronti di più titolari o responsabili del trattamento stabiliti in Stati membri diversi innanzi ai giudici dello Stato membro in cui uno di essi è stabilito - possano operare anche rispetto alle azioni introdotte dai titolari dei dati sulla base dei criteri contemplati dall'art. 79 del regolamento sulla protezione dei dati personali ${ }^{10}$.

\footnotetext{
${ }^{7} \mathrm{Si}$ veda in proposito P. FranzINA, Jurisdiction Regarding Claims for the Infringement of Privacy Rights under the General Data Protection Regulation, in A. De Franceschi (ed.), European Contract Law and the Digital Single Market. The Implications of the Digital Revolution, Cambridge, Antwerp, Portland, 2016, p. 81 ss., spec. p. 103 s.

8 Diversamente, in questo, dalla regola concernente il foro del fatto illecito nel regolamento Bruxelles I-bis, la quale, secondo una logica di neutralità rispetto alle posizioni sostanziali delle parti che è comune anche alle altre regole relative ai fori speciali nel regolamento stesso, si applica anche relativamente ad eventuali azioni di accertamento negativo promosse dal presunto responsabile: si veda, con riferimento alla regola corrispondente contenuta nel precedente regolamento $\mathrm{n}$. 44/2001 ("Bruxelles I"), Corte di giustizia UE, sent. 25 ottobre 2012, in causa C-133/11, Folien Fischer AG c. Ritrama s.p.a., ECLI:EU:C:2012:664, par. 41 ss., massima in Rivista di diritto internazionale privato e processuale, 2012, p. 964 s.; in Revue critique de droit international privé, 2013, p. 501 ss., nota di H. MuIR-WATT, ivi, p. 506 ss.

9 Si veda infra, III.

${ }^{10}$ L'estensione della competenza giurisdizionale del giudice designato in base ai criteri di cui all'art. 79, par. 2, del regolamento n. 2016 /679 alle eventuali chiamate di terzo e domande riconvenzionali spiegate dal titolare ovvero dal responsabile del trattamento non appare infatti suscettibile di pregiudicare l'applicazione di tali criteri, non apportando ad essi alcuna deroga, mentre appare strumentale all'obiettivo di assicurare una tutela giurisdizionale effettiva dei diritti riconosciuti dal regolamento, la quale verrebbe ostacolata da un'eventuale dispersione del contenzioso scaturente dall'invocazione dei diritti riconosciuti dal regolamento da parte del titolare dei dati. Si veda in questo senso anche P. FranzINA, Jurisdiction Regarding Claims for the Infringement of Privacy Rights under the General Data Protection Regulation, cit., p. 103 ss.; più generoso, ma meno con-
} 
7. Più dubbia appare invece la possibilità di invocare, in relazione alle azioni cui si applicano i criteri di giurisdizione posti dal regolamento n. 2016/679, l'applicazione delle disposizioni contenute nel regolamento Bruxelles I-bis in materia di deroga e proroga della giurisdizione. Deve infatti osservarsi che l'applicazione di tali disposizioni è certo suscettibile di pregiudicare in senso formale l'applicazione dei criteri di giurisdizione contenuti nel regolamento sulla protezione dei dati personali, producendo l'effetto di sottoporre le azioni contemplate da tale regolamento a giudici di Stati membri diversi da quelli individuati in base ai criteri fissati dal regolamento stesso. Al tempo stesso, nel momento in cui si ammette, nell'ottica di contribuire all'obiettivo di offrire al titolare dei dati una tutela giurisdizionale più effettiva dei propri diritti, l'integrazione della, a dire il vero, piuttosto scarna disciplina in punto di giurisdizione recata dal regolamento n. 2016/679 con determinati strumenti procedurali, come la connessione attributiva, offerti dalla ordinaria disciplina della giurisdizione in materia civile e commerciale recata dal regolamento Bruxelles I-bis, un'eadem ratio dovrebbe consentire di ammettere la deroga ai criteri di giurisdizione fissati dal regolamento n. 2016/679 nella misura in cui questa operi a favore del titolare dei dati personali. In questi termini, appare potersi ammettere senza troppa difficoltà la proroga tacita contemplata dall'art. 26 del regolamento Bruxelles I-bis, considerato che, come già rilevato, i criteri di giurisdizione contemplati dall'art. 79, par. 2, del regolamento n. 2016/679 si applicano alle sole azioni proposte dal titolare dei dati nei confronti del titolare ovvero del responsabile del trattamento, per cui l'eventuale accettazione da parte di questi ultimi della giurisdizione di un giudice di uno Stato membro diverso da quelli contemplati da quest'ultimo regolamento, che sia stato adito dal titolare dei dati, giungerebbe comunque a beneficio di quest'ultimo, che verrebbe in questo modo dispensato dal dover introdurre una nuova azione innanzi ai giudici di uno Stato membro diverso. Maggiormente problematica si presenta, invece l'ammissibilità di un accordo di scelta del foro ai sensi dell'art. 25 del regolamento Bruxelles I-bis. Un tale accordo rischierebbe, infatti, di venire imposto dal titolare ovvero dal responsabile del trattamento al titolare dei dati, per costringerlo ad accettare la giurisdizione dei giudici di uno Stato membro diverso da quelli indicati nell'art. 79, par. 2, del regolamento n. 2016/679. Come è stato prospettato in dottrina, potrebbe farsi riferimento in proposito alla soluzione accolta nello stesso regolamento "Bruxelles I-bis" nelle materie per le quali, in presenza di un'esigenza di protezione della parte debole di determinati rapporti, come i contratti dei consumatori, i contratti di assicurazione e i contratti individuali di lavoro, esso reca una disciplina più restrittiva in materia di accordi di scelta del foro, volta a renderli ammissibili solamente nella misura in cui consentano alla parte debole del rapporto di adire altri giudici in aggiunta a quelli designati dalle disposizioni del regolamento, affinché il diritto del titolare dei dati di ricorrere ai giudici designati dall'art. 79, par. 2, del regolamento n. 2016/679 non abbia ad esserne pregiudicato ${ }^{11}$.

8. I criteri di giurisdizione contemplati dall'art. 79, par. 2, del regolamento sulla protezione dei dati personali prevedono un'alternativa tra i giudici dello Stato membro in cui il titolare o responsabile del trattamento ha uno stabilimento, e i giudici dello Stato membro in cui il titolare dei dati ha la propria residenza abituale, quest'ultima opzione restando peraltro esclusa nei casi in cui il titolare o responsabile del trattamento sia una pubblica autorità di uno Stato membro, la quale agisca nell'esercizio dei propri pubblici poteri ${ }^{12}$.

divisibile, l'approccio di CH. KoHLer, Conflict of Law Issues in the 2016 Data Protection Regulation of the European Union, in Rivista di diritto internazionale privato e processuale, 2017, p. 653 ss., spec. p. 668 ss., per il quale anche l'applicazione di criteri di giurisdizione non contemplati dal regolamento n. 2016/679 non pregiudicherebbe l'applicazione di questi ultimi.

11 Appare condivisibile in proposito l'analisi svolta da P. FranzINA, Jurisdiction Regarding Claims for the Infringement of Privacy Rights under the General Data Protection Regulation, cit., p. 106 ss.; appare ammettere tale soluzione, più sinteticamente, anche Сн. KoHler, Conflict of Law Issues in the 2016 Data Protection Regulation of the European Union, cit., p. 669 ss., argomentando analogamente che in questo modo l'applicazione dei criteri di giurisdizione contemplati dal regolamento $\mathrm{n}$. 2016/679 non verrebbe ad essere pregiudicata.

12 Si vedano in proposito, tra gli altri, P. DE Miguel Asensio, Aspectos internacionales del Reglamento general de protección de datos de la UE (I): cuestiones de competencia, in www.pedrodemiguelasensio.blogspot.it, 11 maggio 2016, p. 3 s.; P. FranzINA, Jurisdiction Regarding Claims for the Infringement of Privacy Rights under the General Data Protection Regulation, cit., p. 96 ss.; Сн. KoHLer, Conflict of Law Issues in the 2016 Data Protection Regulation of the European Union, cit., p. 668 ss. 
9. In proposito, il primo dei due criteri contemplati dalla norma appare tendenzialmente coincidere, nell'economia di un'azione di carattere extracontrattuale da fatto illecito - come nella gran parte dei casi appare dover essere qualificata un'azione che trova il suo fondamento nella violazione delle disposizioni del regolamento sul trattamento dei dati personali, benché il trattamento dei dati possa materialmente avere luogo anche in occasione della conclusione o in relazione con l'esecuzione di un contratto $^{13}-$ col foro del luogo della condotta ${ }^{14}$, in quanto è normalmente nel luogo in cui il titolare $o$ responsabile del trattamento dei dati è stabilito che il trattamento stesso ha luogo. Pur sempre, deve osservarsi che la norma appare fare riferimento genericamente allo Stato membro in cui il titolare o responsabile del trattamento ha uno stabilimento, senza specificare in proposito che debba trattarsi dello stabilimento principale di tale soggetto - ciò che consentirebbe di accostare tale criterio al foro generale del domicilio del convenuto contemplato dal regolamento Bruxelles I-bis - né che debba trattarsi dello stabilimento presso il quale ha avuto luogo il trattamento dei dati personali che ha dato origine all'azione in giudizio, ciò che consentirebbe più propriamente di assimilare tale foro al foro del luogo della condotta dannosa, secondo l'interpretazione c.d. ubiquitaria che è stata data dalla Corte di giustizia al foro contemplato dall'attuale art. 7, par. 2, del regolamento Bruxelles I-bis nella giurisprudenza della Corte di giustizia relativa agli illeciti a distanza ${ }^{15}$. Piuttosto, nei termini vaghi e generici nei quali è concepito il foro in questione nel regolamento sul trattamento dei dati personali, esso appare assimilabile a un foro basato sulla mera presenza commerciale, la cui portata va ben al di là del foro dell'agenzia, succursale o filiale contemplato dal regolamento Bruxelles I-bis. Quest'ultimo foro, come è noto, oltre a presupporre che il convenuto sia domiciliato in uno Stato membro, è invocabile unicamente con riferimento alle azioni che traggano il loro fondamento dalle attività dell'agenzia, succursale o filiale in questione ${ }^{16}$.

\section{(B) L'applicabilità delle regole di giurisdizione del regolamento $\mathrm{n}$. 2016/679 nei confronti di titola- ri o responsabili del trattamento stabiliti in paesi terzi.}

10. Nella sua ampiezza, il foro contemplato dal regolamento n. 2016/679 appare invocabile anche nei confronti di un titolare o responsabile del trattamento che abbia il proprio stabilimento principale

${ }^{13}$ L'azione nei confronti del titolare o del responsabile del trattamento potrebbe presentare carattere contrattuale ove il trattamento dei dati formasse specificamente oggetto del contratto o di sue clausole. Si veda in proposito CH. KoHLER, Conflict of Law Issues in the 2016 Data Protection Regulation, cit., p. 669 s., 671 s., il quale prospetta l'applicazione alternativa dei criteri recati, rispettivamente, dall'art. 7.1 e 7.2 del regolamento n. 1215/2012 ("Bruxelles I-bis"), sulla base di una lettura restrittiva della clausola di cui all'art. 67 di quest'ultimo regolamento. Secondo l'interpretazione che appare più corretta, invece, il regolamento Bruxelles I-bis, nel dichiarare all'art. 67 di non voler pregiudicare l'applicazione delle disposizioni che disciplinano la competenza giurisdizionale in materie particolari, contenute in atti dell'Unione europea o in legislazioni nazionali armonizzate in applicazione di tali atti, esclude la possibilità di fare ricorso ai propri criteri di competenza giurisdizionale relativamente ad azioni per le quali un altro atto dell'Unione preveda una diversa allocazione della competenza giurisdizionale in considerazione dei caratteri specifici delle controversie contemplate da tale atto. Si vedano, in quest'ultimo senso, P. DE Miguel Asensio, Aspectos internacionales del Reglamento general de protección de datos, cit., p. 3; P. FranzInA, Jurisdiction Regarding Claims for the Infringement of Privacy Rights, cit., p. 105.

14 Secondo l'interpretazione del criterio di competenza giurisdizionale come al tempo contenuto nell'art. 5, n. 3, della Convenzione di Bruxelles del 1968 data dalla Corte di giustizia CE, sent. 30 novembre 1976, in causa 21/76, Bier c. Mines de potasse d'Alsace, in Raccolta, 1976, p. 1735 ss., punti 13 ss. della motivazione, nei casi di illeciti c.d. a distanza, in cui il luogo della condotta illecita è situato in uno Stato membro diverso da quello di verificazione dell'evento dannoso, l'azione potrà proporsi alternativamente nell'uno come nell'altro foro: si vedano in proposito per tutti, A. DAvì, La responsabilità extracontrattuale nel nuovo diritto internazionale privato italiano, Torino, 1997, p. 108 ss.; K. Kerameus, La compétence internationale en matière delictuelle dans la Convention de Bruxelles, in Travaux du Comité français de droit international privé, 1992-1993, Paris, 1994, p. 255 ss., spec. p. 257 ss.; L. MARI, Il diritto processuale civile della convenzione di Bruxelles, I, Il sistema della competenza, Padova, 1999, p. 388 ss.; con riferimento all'incidenza della prevedibilità della competenza giurisdizionale sul diritto delle parti alla tutela giurisdizionale si rinvia a F. MARONGIU BUONAIUTI, La tutela del diritto di accesso alla giustizia e della parità delle armi tra i litiganti nella proposta di revisione del regolamento $n$. 44/2001, in A. Di STEFANO, R. SAPIENZA (a cura di), La tutela dei diritti umani e il diritto internazionale, XVI Convegno SIDI, Catania, 23-24 giugno 2011, Napoli, 2012, p. 345 ss., spec. p. 348 ss.

15 Con particolare riferimento alla sent. 30 novembre 1976, Bier c. Mines de potasse d'Alsace, cit. (nota prec.).

16 Si veda in proposito P. Franzina, Jurisdiction Regarding Claims for the Infringement of Privacy Rights, cit., p. 99 ss., sottolineando la chiara finalità protettiva nei confronti del titolare dei dati insita nella previsione di un così ampio criterio di competenza giurisdizionale. 
in uno Stato terzo, qualora abbia uno stabilimento nello Stato membro del giudice adito, e ciò anche qualora il trattamento dei dati oggetto della domanda giudiziale non rientri nell'ambito delle attività di tale stabilimento, ma a condizione, al tempo stesso, che il diritto la cui tutela è invocata dal titolare dei dati ricada nell'ambito di applicazione ratione loci vel personarum del regolamento stesso. In proposito, ai sensi dell'art. 3 del regolamento, la presenza di uno stabilimento del titolare o responsabile del trattamento in uno Stato membro è sufficiente al fine dell'applicazione delle disposizioni del regolamento stesso al trattamento di dati che sia effettuato nel contesto delle attività di quello stabilimento. Ciò, in definitiva, nelle ipotesi di un titolare o responsabile del trattamento il cui stabilimento principale sia situato in uno Stato terzo e che abbia uno stabilimento in uno Stato membro, consente di invocare il foro in questione relativamente alle sole azioni scaturenti dal trattamento dei dati che sia effettuato nel contesto delle attività di tale stabilimento.

11. La norma dell'art. 3, par. 1, del regolamento, a questo riguardo, appare recepire l'interpretazione senz'altro ampia dell' ambito di applicazione ratione personarum della previgente disciplina europea del trattamento dei dati personali fatta propria dalla Corte di giustizia nella sentenza Google Spain, precisando che la disciplina contenuta nel regolamento si applica indipendentemente dal fatto che il trattamento dei dati sia materialmente avvenuto all'interno dell'Unione o meno, essendo sufficiente che esso sia imputabile a uno stabilimento del titolare o responsabile del trattamento che sia ubicato nell'Unione ${ }^{17}$. Del resto, nel caso, a cui si riferiva la sentenza appena evocata, in cui il trattamento dei dati sia effettuato da un gestore di un sito Internet, non è certo infrequente che quest'ultimo possa materialmente delocalizzare le operazioni relative al trattamento dei dati degli utenti, eventualmente affidandole a un soggetto terzo ubicato in un paese che non presenta alcun effettivo collegamento con la vicenda che ha dato luogo all'acquisizione dei dati. Conseguentemente, al fine dell'applicazione ratione personarum della disciplina recata dal regolamento, e, conseguentemente, dell'invocabilità del criterio di giurisdizione accolto nel suo art. 79, par. 2 nei confronti di un titolare o di un responsabile del trattamento il cui stabilimento principale sia in uno Stato terzo, può rilevare unicamente, anche a fini di certezza del diritto e di prevedibilità della competenza giurisdizionale e dell'applicabilità stessa della disciplina recata dal regolamento in esame, lo stabilimento sito in uno Stato membro nel contesto delle attività del quale i dati in questione sono stati acquisiti ${ }^{18}$.

12. In un'ottica ulteriormente estensiva dell'ambito di applicazione soggettivo del regolamento, ai sensi dell'art. 3, par. 2, le sue norme, e di conseguenza i criteri di competenza giurisdizionale contemplati dall'art. 79, par. 2, quantomeno relativamente al foro della residenza abituale del titolare dei dati, sono invocabili anche nei confronti di un titolare o responsabile del trattamento che non abbia alcun stabilimento in uno Stato membro, ogniqualvolta i dati oggetto del trattamento si riferiscano a persone che si trovino materialmente in uno Stato membro. A questo fine, però, all'evidente scopo di tutelare il titolare ovvero responsabile del trattamento, stabilito in un paese terzo, da un'applicazione "a sorpresa" della disciplina recata dal regolamento e di contenere in qualche misura la tendenza all'applicazione extraterritoriale della disciplina protettiva da esso recata, l'art. 3, par. 2, pone alcuni requisiti ulteriori, atti a garantire l'esistenza di un collegamento oggettivo e prevedibile del trattamento dei dati con lo Stato membro in cui il titolare dei dati stessi si trova. Tali requisiti ulteriori sono individuati dalla norma

17 Corte di giustizia UE, sent. 13 maggio 2014, in causa C-131/12, Google Spain SL, Google Inc, ECLI:EU:C:2014:317. Nel senso di un'interpretazione estensiva della nozione di trattamento dei dati che intervenga nel contesto delle attività di uno stabilimento del responsabile del trattamento nell'Unione, ai fini dei corrispondenti criteri di applicazione territoriale della disciplina contenuta nella precedente direttiva 95/46/CE, si veda anche Corte di giustizia UE, sent. 1 ottobre 2015, in causa C-230/14, Weltimmo, ECLI:EU:C:2015:639, in Revue critique de droit international privé, 2016, p. 377 ss., con nota di B. Haftel, ivi, p. 378 ss.; v. anche CH. Kohler, Conflict of Law Issues in the 2016 Data Protection Regulation, cit., p. 658 ss.

$18 \mathrm{Si}$ veda ancora CH. KoHLer, Conflict of Law Issues in the 2016 Data Protection Regulation, cit., p. 659 s. Diversamente, ai fini del criterio di competenza giurisdizionale contemplato dall'art. 79, par. 2, del regolamento n. 2016/679, la genericità del riferimento a "uno stabilimento" del responsabile del trattamento e la ratio consistente nell'obiettivo di assicurare al titolare dei dati le più ampie prospettive di accesso a un giudice innanzi al quale poter agire nei confronti del responsabile del trattamento suggeriscono un'interpretazione più ampia: v. P. FranzINA, Jurisdiction Regarding Claims for the Infringement of Privacy Rights, cit., p. $100 \mathrm{~s}$. 
nell'essere il trattamento dei dati legato alternativamente all'offerta di beni o servizi a titolari dei dati che si trovino nell'Unione, ovvero al monitoraggio del loro comportamento, nella misura in cui il comportamento oggetto del monitoraggio abbia luogo nell'Unione.

13. Infine, deve rilevarsi che il regolamento, all'art. 3, par. 3, tende a superare i normali limiti territoriali dell'applicazione del diritto dell'Unione, per i quali questo di regola non si applica nei territori extraeuropei soggetti alla sovranità degli Stati membri, prevedendo l'applicazione delle proprie norme ai titolari o responsabili del trattamento che siano stabiliti in un luogo soggetto al diritto di uno Stato membro in base al diritto internazionale ${ }^{19}$.

(C) l'opzione consentita dal regolamento a favore del foro della residenza abituale del titolare dei dati personali e le sue criticità, alla luce della parallela giurisprudenza della Corte di giustizia relativa alle violazioni della privacy e dei diritti della personalità.

14. Sussistendo le condizioni che ne determinano l'assoggettamento alla disciplina recata dal regolamento n. 2016/679 che si sono evidenziate, il titolare o il responsabile del trattamento potrà essere citato, in alternativa allo Stato membro in cui ha un proprio stabilimento nel senso che si è indicato, innanzi ai giudici dello Stato membro in cui il titolare dei dati ha la propria residenza abituale, salvo che, come già si è menzionato, il titolare o responsabile del trattamento sia una pubblica autorità che agisca nell'esercizio dei propri pubblici poteri, ciò che, per definizione, ne rende difficilmente configurabile l'assoggettamento alla giurisdizione dei giudici di uno Stato diverso. Il criterio alternativo costituito dalla residenza abituale del titolare dei dati presenta un'innegabile assonanza col criterio del centro degli interessi della persona che si pretenda vittima di una violazione della privacy o di altro diritto della personalità, utilizzato dalla Corte di giustizia nella sentenza $e$ Date relativamente alle violazioni di tali diritti commesse tramite informazioni pubblicate su siti Internet e localizzato tendenzialmente nel luogo in cui la persona che si pretende lesa ha per l'appunto la propria residenza abituale ${ }^{20}$, e si rivela atto a coincidere tendenzialmente col giudice del luogo dell'eventum damni in un'azione risarcitoria da fatto illecito.

15. Ciò può trovare giustificazione alla luce della considerazione che la violazione dei diritti conferiti dal regolamento $\mathrm{n}$. 2016/679 al titolare dei dati personali, in quanto atta a colpire la persona del titolare dei dati in un suo diritto della personalità quale è quello al controllo dei propri dati personali, deve considerarsi materializzata, quale luogo dell'evento dannoso, nel luogo in cui la persona è stabilita, che il regolamento, con una soluzione che è ormai ampiamente accolta nella generalità degli atti dell'Unione europea in materia di diritto internazionale privato, identifica nella residenza abituale del

\footnotetext{
19 Secondo quanto affermato nel considerando n. 25 del preambolo del regolamento, in base a questa disposizione le norme del regolamento potrebbero trovare applicazione a un responsabile del trattamento che sia stabilito all'interno di una rappresentanza diplomatica o posto consolare di un paese membro ubicati in un paese terzo, ipotesi abbastanza singolare ove non la si intenda riferire al trattamento dei dati personali effettuato dagli uffici stessi della rappresentanza diplomatica o del posto consolare per l'esercizio delle loro funzioni. Si veda in proposito CH. KoHLER, Conflict of Law Issues in the 2016 Data Protection Regulation, cit., p. 660, il quale rileva che l'ambito di applicazione del regolamento si estende fino ai limiti più esterni della giurisdizione statale consentiti dal diritto internazionale.

${ }^{20}$ Corte di giustizia UE, sent. 25 ottobre 2011, cause riunite C-509/09 e C-161/10, e-Date Advertising GmbH c. X, Martinez c. MGN Ltd, in Raccolta, 2011, p. I-10269 ss.; in proposito, si vedano O. FerACI, Diffamazione internazionale a mezzo di Internet: quale foro competente? Alcune considerazioni sulla sentenza eDate, in Rivista di diritto internazionale, 2012, p. 461 ss.; G. GuIzIOU, nota in Journal du droit international, 2012, p. 201 ss.; S. MARINo, La violazione dei diritti della personalità nella cooperazione giudiziaria civile europea, in Rivista di diritto internazionale privato e processuale, 2012, p. 363 SS.; E. GABELLINI, La competenza giurisdizionale nel caso di lesione di un diritto della personalità attraverso Internet, in Rivista trimestrale di diritto e procedura civile, 2014, p. 271 ss.; si rimanda anche a F. MARONGIU BUONAIUTI, Le obbligazioni non contrattuali nel diritto internazionale privato, Milano, 2013, p. 26 ss. Si vedano anche, da ultimo, nel senso di ritenere applicabile, con alcuni temperamenti, la soluzione accolta nel caso eDate nell'ipotesi in cui a lamentare una lesione dell'onore o della reputazione per effetto di notizie pubblicate su un sito Internet sia una persona giuridica e l'azione di questa sia volta principalmente ad ottenere la rimozione delle notizie diffamatorie dal sito ospitante, le conclusioni dell'Avvocato generale Bobek, presentate il 13 luglio 2017, relativamente alla causa C-194/16, Bolagsupplysningen OÜ c. Svensk Handel AB, ECLI:EU:C:2017:554, su cui A. BIzER, Opinion of Advocate General Bobek on jurisdiction in cases concerning violations of personality rights on the Internet (Bolagsupplysningen, C-194/16), scritto pubblicato su www.conflictoflaws.net, 3 agosto 2017.
} 
soggetto $^{21}$. Si deve nondimeno sottolineare che la stessa Corte di giustizia nella sentenza eDate ha avuto modo di precisare che la coincidenza del centro degli interessi della persona che si pretende lesa dalla violazione dei propri diritti della personalità per effetto della pubblicazione di informazioni su un sito Internet con la sua residenza abituale non costituisce un dato irrefutabile, ben potendo tale centro degli interessi essere collocato, a seconda delle circostanze, nel luogo di prevalente localizzazione dell'attività professionale del soggetto che si pretende leso, il quale potrebbe essere ubicato in un paese membro diverso da quello della residenza abituale ${ }^{22}$.

16. Inevitabilmente, l'opzione che l'art. 79, par. 2, del regolamento n. 2016/679 prevede a favore del foro dello Stato membro della residenza abituale del titolare dei dati si presta alla medesima obiezione che è stata rivolta al foro del centro degli interessi della persona che si pretenda vittima di una violazione della privacy o di altro diritto della personalità, contemplato dalla Corte di giustizia nell'interpretazione del criterio speciale oggi contenuto nell'art. 7, par. 2, del regolamento n. 1215/2012 accolta nella sentenza eDate. Tale obiezione riguarda il rischio di pregiudicare eccessivamente, a favore del soggetto che si pretenda leso, la parità delle armi tra i litiganti, che è parte integrante del diritto all'equo processo tutelato dall'art. 6, par. 1, della Convenzione europea dei diritti dell'uomo e, trattandosi dell'applicazione, nell'un caso come nell'altro, di un atto dell'Unione europea, dall'art. 47 della Carta dei diritti fondamentali ${ }^{23}$. A questo proposito, possono venire in considerazione le giustificazioni già addotte dalla Corte di giustizia nella sentenza appena evocata, le quali si incentravano essenzialmente sulla particolare attitudine pregiudizievole della diffusione di informazioni tramite Internet, attesa la grande facilità ed immediatezza con la quale esse possono essere consultate da utenti situati in diverse parti del mondo ${ }^{24}$.

17. Queste considerazioni, peraltro, sono trasponibili solo in parte al contesto della tutela dei dati personali apprestata dal regolamento n. 2016/679, considerato che questa presenta una portata generale ed è pertanto destinata ad applicarsi indipendentemente dal mezzo attraverso il quale possa essere arrecata una lesione ai diritti che il regolamento conferisce al titolare dei dati. Inoltre, deve rilevarsi che la scelta che è offerta al titolare dei dati - e a lui soltanto data l'ottica nella quale è concepita la norma dell'art. 79, par. 2, del regolamento - tra due possibili fori alternativi, dei quali il secondo presenta un evidente legame di stretta prossimità con la sua personale sfera giuridica, si inserisce pienamente nella

${ }^{21} \mathrm{Si}$ veda in proposito P. Franzina, Jurisdiction Regarding Claims for the Infringement of Privacy Rights, cit., p. 101 ss. Nel senso per cui, problematicamente, il criterio in questione potrebbe coesistere col criterio del centro degli interessi del titolare dei dati derivante dall'art. 7.2 del regolamento Bruxelles I-bis secondo l'interpretazione datane nella sentenza eDate, per cui tale centro potrebbe anche materialmente non coincidere ed essere potenzialmente ubicato in un paese membro diverso da quello della residenza abituale del soggetto in questione, Сн. KoHLER, Conflict of Law Issues in the 2016 Data Protection Regulation, cit., p. 669 s.

${ }^{22}$ Corte di giustizia UE, sent. 25 ottobre 2011, e-Date Advertising GmbH c. X, Martinez c. MGN Ltd, cit., par. 49. Si vedano ulteriormente, quanto alla problematica insita nella localizzazione del centro degli interessi della persona che si pretende lesa da una violazione del proprio diritto all'onore o alla reputazione commessa a mezzo di informazioni pubblicate su un sito Internet, con particolare, ma non esclusivo, riferimento all'ipotesi in cui il soggetto che si pretende leso sia una persona giuridica, le conclusioni dell'Avvocato generale Bobek del 13 luglio 2017 relative alla causa Bolagsupplysningen OÜ c. Svensk Handel $A B$, cit., par. 99 ss.

23 Si rimanda alle considerazioni svolte in F. MArongiu BuonaIuTI, La tutela del diritto di accesso alla giustizia e della parità delle armi tra i litiganti, cit., p. 348 ss.

${ }^{24}$ Corte di giustizia UE, sent. 25 ottobre 2011, e-Date Advertising GmbH c. X, Martinez c. MGN Ltd, cit., par. 45 ss.; si vedano anche, sulla scia della sentenza eDate, le conclusioni dell'Avvocato generale Bobek del 13 luglio 2017 relative alla causa Bolagsupplysningen OÜ c. Svensk Handel AB, cit., par. 74 ss., nelle quali l'Avvocato generale, nel confermare la pertinenza del criterio del centro degli interessi della persona che si pretende lesa da una violazione dei propri diritti della personalità per effetto di informazioni pubblicate tramite Internet, propone nondimeno un ridimensionamento dell'ampiezza del ventaglio di fori alternativi offerto alla persona che si pretende lesa da una tale violazione, propendendo per l'esclusione della soluzione basata sulla c.d. Mosaikbetrachtung, che era stata concepita nella precedente sentenza della Corte di giustizia del 7 marzo 1995, in causa C-68/93, Shevill c. Presse Alliance SA, in Raccolta, 1995, p. I-415 ss., in relazione a casi di diffamazione a mezzo stampa, per la quale in alternativa ai giudici del luogo in cui ha sede l'editore della pubblicazione diffamatoria il soggetto leso avrebbe potuto agire anche negli ulteriori Stati membri in cui la pubblicazione sia stata diffusa, limitatamente ai danni verificatisi in tali Stati membri. Ciò in quanto, in relazione ad informazioni pubblicate su un sito Internet, è difficilmente praticabile un controllo attendibile della diffusione delle informazioni pubblicate in paesi membri diversi ed è similmente difficile una conseguente quantificazione dei danni prodottisi per effetto di tale diffusione in un paese piuttosto che in un altro. 
logica d'insieme del regolamento, consistente nel perseguimento dell'obiettivo di garantire un elevato livello di tutela dei diritti del titolare dei dati ${ }^{25}$. In proposito, oltre al rilievo per il quale la norma in questione si presenta espressamente come una specificazione delle modalità di attuazione del diritto ad un rimedio giurisdizionale effettivo che è riconosciuto al titolare del dati dal par. 1 della stessa disposizione dell'art. 79 del regolamento, deve essere rilevato come l'ampiezza della tutela offerta sul piano processuale dalla norma in esame riflette l'ampiezza della tutela che al titolare dei dati è offerta sul piano sostanziale dalla disciplina uniforme che il regolamento stesso introduce.

18. Particolarmente indicativa di tale ampiezza si presenta la disposizione di cui all'art. 82 del regolamento n. 2016/679, la quale prevede, al par. 1, il diritto del titolare dei dati al risarcimento dei danni materiali e immateriali da parte del titolare ovvero del responsabile del trattamento dei dati, dettando ai paragrafi successivi un regime autonomo di responsabilità relativamente a tali soggetti. In particolare, il par. 2 della norma contempla un regime particolarmente rigoroso per il primo di questi due soggetti, qualificabile in termini di responsabilità oggettiva, prevedendo che il titolare del trattamento risponda del danno cagionato al titolare dei dati personali per effetto di un trattamento dei dati in modalità non conforme a quanto previsto dal regolamento, laddove un regime più ristretto è previsto dalla disposizione in esame per l'insorgere della responsabilità del responsabile del trattamento, il quale potrà essere chiamato a rispondere per i danni causati dal trattamento dei dati personali unicamente in presenza di violazioni di obblighi specificamente posti dal regolamento in capo ai responsabili del trattamento ovvero in caso di violazione di istruzioni specificamente date dal titolare dei dati in ordine al trattamento dei dati stessi. Ugualmente nell'ottica, espressamente dichiarata nel par. 4 della norma, di garantire al titolare dei dati un effettivo ristoro dei danni subiti in conseguenza di violazioni della disciplina posta dal regolamento, i successivi paragrafi della norma pongono a carico del titolare ovvero del responsabile del trattamento l'onere di provare che l'evento dannoso non è a loro imputabile, e stabiliscono un regime di responsabilità solidale per i casi di violazioni imputabili a una pluralità di titolari ovvero di responsabili del trattamento, oppure a uno o più titolari e a uno o più responsabili del trattamento allo stesso tempo ${ }^{26}$.

\section{(D) l'imperatività della tutela del titolare dei dati come giustificazione sistematica dell'opzione a favore del foro della sua residenza abituale.}

19. La particolare imperatività della tutela del titolare dei dati personali che ispira la disciplina recata dal regolamento è ulteriormente sottolineata, tra l'altro, dai rigidi limiti che il regolamento stesso pone alla possibilità per un titolare o responsabile del trattamento, la cui attività ricada nell' ambito territoriale ovvero personale di applicazione della disciplina da questo recata, di sottrarsi alla sua applicazione. Rileva in questo senso particolarmente la disposizione dell'art. 48 del regolamento, la quale, con un approccio che si rivela peraltro eccessivamente rigido e massimalista, esclude il riconoscimento di decisioni giudiziarie o adottate da autorità amministrative di Stati terzi, che richieda al titolare o al responsabile del trattamento di trasferire o rivelare dati personali, in assenza di un accordo internazionale in vigore tra lo Stato in questione e l'Unione europea o un suo Stato membro, salvo che il regolamento stesso disponga diversamente.

20. La norma, che sottolinea il carattere anche internazionalmente imperativo della disciplina recata dal regolamento, in quanto non consente che la sua applicazione possa essere esclusa, relativamente a situazioni ricadenti nel suo ambito di applicazione, per effetto dell'applicazione di una disciplina potenzialmente non corrispondente da parte di un giudice di un paese terzo ${ }^{27}$, appare peraltro criti-

${ }^{25}$ Secondo quanto osservato anche da P. Franzina, Jurisdiction Regarding Claims for the Infringement of Privacy Rights, cit., p. $97 \mathrm{~s}$.

${ }^{26}$ Si veda in proposito Сн. KoHLer, Conflict of Law Issues in the 2016 Data Protection Regulation, cit., p. 672.

$27 \mathrm{Si}$ veda ancora CH. KohleR, Conflict of Law Issues in the 2016 Data Protection Regulation, cit., p. 661 s., con riferimento a un'affermazione in questo senso, riferita alla precedente disciplina di cui alla direttiva 95/46/CE, contenuta nella già citata sentenza della Corte di giustizia UE, 13 maggio 2014, in causa C-131/12, Google Spain SL, Google Inc, cit., par. 58, nonché all'affermazione da parte della Corte del carattere internazionalmente imperativo della disciplina comunitaria protettiva dei 
cabile nell'approccio adottato. Infatti, facendo riferimento unicamente al dato formale della presenza o meno di un accordo con lo Stato terzo da cui la decisione provenga, non attribuisce alcuna rilevanza agli standards di tutela dei dati personali in vigore nello Stato terzo in questione, ovvero previsti dalla legge di cui i giudici di tale Stato abbiano fatto applicazione, che potrebbero non necessariamente rivelarsi deteriori rispetto a quelli previsti dal regolamento, a prescindere dall'esistenza di alcun accordo con lo Stato terzo che viene in considerazione.

\section{Le disposizioni concernenti la sospensione delle azioni in caso di procedimenti paralleli, in rapporto alla disciplina in materia di litispendenza e connessione c.d. privativa nel regolamento "Bruxelles I-bis".}

21. Il regolamento n. 2016/679, oltre a dettare proprie specifiche regole di giurisdizione per le azioni introdotte dai titolari dei dati personali nei confronti dei titolari ovvero dei responsabili del trattamento, prevede, nello stesso capitolo VIII, proprie apposite disposizioni sul coordinamento tra azioni parallele che possano essere introdotte in base alle regole di giurisdizione contenute nel regolamento stesso. Non diversamente da queste ultime regole, anche le norme concernenti il coordinamento tra azioni parallele riflettono in larga parte, pur in assenza, anche in questo caso, di alcun riferimento espresso, il modello offerto dalle regole in materia di litispendenza e di connessione privativa contenute nel regolamento n. 1215/2012 o "Bruxelles I-bis", e già, salve alcune varianti, nel previgente regolamento n. 44/2001 o "Bruxelles I" ed ancor prima nella Convenzione di Bruxelles del $1968^{28}$.

22. Anche in questo caso, la presenza di specifiche regole in materia all'interno del regolamento n. 2016/679 appare precludere, conformemente alla regola generale contenuta nell'art. 67 del regolamento "Bruxelles I-bis" e richiamata, come già rilevato, nel considerando n. 147 del preambolo del regolamento in esame, l'applicazione delle omologhe disposizioni contenute nel regolamento $\mathrm{n}$. $1215 / 2012^{29}$. Pur sempre, se con riferimento alle regole di giurisdizione propriamente intese la non piena corrispondenza delle regole contenute nel regolamento n. 2016/679 alle regole proprie del regolamento "Bruxelles I-bis", nell'interpretazione che ne è stata data dalla Corte di giustizia, può trovare giustifica-

diritti degli agenti commerciali indipendenti contenuta nella nota sentenza del 9 novembre 2000, in causa C-381/98, Ingmar GB Ltd c. Eaton Leonard Technologies Inc., in Raccolta, 2000, p. I-9305 ss., par. 25.

${ }_{28}$ Sulle quali ci si permette di rinviare a F. Marongiu BuonaIuti, Litispendenza e connessione internazionale. Strumenti di coordinamento tra giurisdizioni statali in materia civile, Napoli, 2008, p. 110 ss.; ID., Lis pendens and Related Actions in Civil and Commercial Matters Within the European Judicial Area, in Yearbook of Private International Law, vol. 11 (2009), p. 511 ss., spec. p. 517 ss. e, per un sintetico quadro delle modifiche introdotte in proposito dal regolamento n. 1215/2012 o "Bruxelles I-bis", ID., Per una prima lettura del regolamento «Bruxelles I-bis»: il regime della litispendenza e della connessione privativa, scritto pubblicato il 19 dicembre 2012 su http://aldricus.com.

29 Si vedano, per una diversa interpretazione che fa leva, tra l'altro, sul considerando n. 144 del preambolo del regolamento n. 2016/679, P. De Miguel Asensio, Aspectos internacionales del Reglamento general de protección de datos de la UE (I): cuestiones de competencia, cit., p. 4; P. FranzINA, Jurisdiction Regarding Claims for the Infringement of Privacy Rights, cit., p. $105 \mathrm{ss}$. In proposito, il considerando in questione, nel fare riferimento ad un'autorità giurisdizionale che sia adita di un'azione esperita nei confronti di una decisione di un'autorità di controllo, appare presentare un difetto di coordinamento col testo del regolamento, dal momento che l'art. 81 , nei termini generali in cui è formulato e nella finalità, che con evidenza persegue, di evitare lo svolgimento di procedimenti paralleli, non appare giustificare una limitazione della sua portata ai soli casi in cui il giudice successivamente adito sia investito di un'azione o, piuttosto, di un ricorso avverso una decisione sfavorevole di un'autorità nazionale di controllo. Inevitabilmente, ed indipendentemente dall'indicazione più restrittiva che appare emergere dal considerando $\mathrm{n}$. 144 del preambolo - il quale, in quanto sprovvisto di efficacia vincolante, non è atto a prevalere sull'ampiezza della formulazione adottata nell'art. 81 del regolamento - il problema del coordinamento tra procedimenti paralleli pendenti innanzi a giudici di Stati membri diversi appare suscettibile di porsi altrettanto, ed anzi più verosimilmente, nei rapporti tra azioni parallele nei confronti di un titolare o responsabile del trattamento. Peraltro, relativamente alle azioni avverso decisioni delle autorità nazionali di controllo il regolamento stesso, all'art. 78, par. 3, prevede una competenza funzionale dei giudici dello Stato membro in cui l'autorità siede, ed è quindi difficile che azioni aventi lo stesso oggetto relativamente al trattamento dei dati da parte del medesimo titolare o responsabile del trattamento, come richiesto dalla norma, possano pendere davanti a giudici di uno Stato membro diverso da quello in cui la decisione dell'autorità di controllo è impugnata, posto che, per definizione, l'oggetto del giudizio di impugnazione di una decisione dell'autorità di controllo è da identificarsi con la legittimità della decisione stessa, che non può che essere valutata dai giudici dello Stato membro in cui l'autorità che la ha emessa ha sede. 
zione, come si è rilevato, nella specifica finalità perseguita dal regolamento sulla tutela dei dati personali di garantire quanto più ampiamente al titolare dei dati personali l'accesso ad una tutela giurisdizionale effettiva dei diritti riconosciutigli dal regolamento stesso $^{30}$, la medesima ratio non appare giustificare altrettanto adeguatamente l'adozione di disposizioni specifiche in punto di coordinamento tra procedimenti paralleli. Queste appaiono, ad un più attento esame, discostarsi sotto diversi aspetti, rivelando per di più una certa imprecisione nei termini in cui sono redatte, dal modello offerto dalle disposizioni contenute allo stesso fine nel regolamento "Bruxelles I-bis", il nucleo essenziale delle quali, tratto dal precedente regolamento "Bruxelles I", figura recepito in termini corrispondenti anche all'interno di altri regolamenti adottati in diversi ambiti nel quadro della cooperazione giudiziaria in materia civile ${ }^{31}$.

23. In concreto, i profili di differenziazione della disciplina del coordinamento tra azioni parallele contenuta nel regolamento n. 2016/679 rispetto al modello offerto dal regolamento "Bruxelles I-bis" ovvero dal previgente regolamento "Bruxelles I" non appaiono affatto trascurabili e possono fare legittimamente sorgere qualche dubbio in ordine alla effettiva rispondenza di tali differenziazioni, suscettibili di tradursi in incertezze e difficoltà di ordine pratico per i giudici chiamati ad applicare le regole in questione, alla già richiamata finalità di garantire una tutela giurisdizionale effettiva dei diritti dei titolari dei dati protetti dal regolamento. Essenzialmente, come è noto, il regolamento "Bruxelles I-bis", come già il precedente regolamento "Bruxelles I" e i regolamenti che in proposito ne hanno seguito il modello - il regolamento n. 4/2009 in materia di obbligazioni alimentari, il regolamento n. 650/2012 in materia di successioni, i regolamenti n. 2016/1103 e 2016/1104 in materia, rispettivamente, di regimi patrimoniali tra coniugi e di effetti patrimoniali delle unioni registrate ${ }^{32}$, una soluzione lievemente diversa essendo invece seguita dal regolamento n. 2201/2003 o "Bruxelles II-bis" in materia matrimoniale e di potestà genitoriale ${ }^{33}$ - distinguono, in materia di coordinamento tra procedimenti paralleli, le due ipotesi della litispendenza e della connessione. Di queste, la prima, benché con la flessibilità fatta propria dalla Corte di giustizia nella propria giurisprudenza ${ }^{34}$, presuppone una completa identità delle due domande, basata sulla comunanza di oggetto, di titolo e di parti, mentre la seconda presuppone che tra le due azioni, benché non identiche, sussistano degli elementi comuni, atti a far sorgere l'opportunità di una trattazione e

30 Si rimanda a quanto osservato supra, II.B.

31 Appare condivisibile in proposito quanto osservato da P. DE Miguel AsEnsio, Aspectos internacionales del Reglamento general de protección de datos de la UE (I): cuestiones de competencia, p. 3 s. e da P. FRANZINA, Jurisdiction Regarding Claims for the Infringement of Privacy Rights, cit., p. 105 ss., nel senso che in presenza di regole sul coordinamento tra procedimenti paralleli ampiamente conosciute dai professionisti e dai giudici dei paesi membri, come quelle contenute attualmente nel regolamento "Bruxelles I-bis", non appare come una scelta opportuna quella compiuta dal legislatore dell'Unione di affiancare ad esse regole ulteriori, formulate per di più in termini meno precisi di quelle contenute in quest'ultimo regolamento.

32 Si veda, nel senso dell'emersione di un corpus di regole comuni su questioni generali attinenti all'applicazione delle regole di giurisdizione, le quali sono presenti in termini corrispondenti nei diversi atti adottati nei vari ambiti ricadenti nel settore della cooperazione giudiziaria in materia civile, H. GAUDEMET-TALLON, Les règles de compétence judiciaire dans le règlement européen sur les successions, in G. KhaIRALlah, M. REVILlarD (eds), Droit européen des successions internationales. Le règlement du 4 juillet 2012, Paris, 2013, p. 127 ss., spec. p. 136, la quale le riunisce sotto la definizione di "règles procédurales concernant la compétence".

33 In proposito, l'art. 19 del regolamento n. 2201/2003 o "Bruxelles II-bis" reca una disciplina distinta dei presupposti della litispendenza per i procedimenti in materia matrimoniale, relativamente ai quali sono equiparati ai fini della litispendenza i procedimenti di separazione personale, di divorzio e di annullamento del matrimonio relativi ai medesimi coniugi, fenomeno icasticamente definito nella relazione esplicativa della Convenzione detta di "Bruxelles II" che è all'origine della disciplina contenuta nel regolamento, redatta da A. Borrás, in G.U.C.E., C 221 del 16 luglio 1998, p. 27 ss., par. 54, come “falsa litispendenza", e per i procedimenti in materia di potestà genitoriale, relativamente ai quali ai fini della litispendenza è richiesta l'identità di oggetto e di titolo e il trattarsi di procedimenti relativi alla protezione del medesimo minore. In considerazione della maggiore ampiezza dei presupposti della litispendenza contemplati relativamente alle due materie oggetto del regolamento, questo non reca una disposizione autonoma relativa alla connessione privativa analoga a quella presente negli altri regolamenti.

34 A cominciare dalle ben note sentenze dell' 8 dicembre 1987, in causa 144/86, Gubisch Maschinenfabrik c. Palumbo, in Raccolta, 1987, p. 4861 ss. e del 6 dicembre 1994, in causa C-406/92, Tatry, in Raccolta, 1994, p. I-5439 ss., nelle quali la Corte di giustizia aveva ritenuto necessario dare un'interpretazione ampia dei presupposti dell'identità di oggetto e di titolo delle due domande allo scopo di assicurare nella maggior misura del possibile la prevenzione del contrasto tra giudicati, da identificarsi come finalità principe della regola in materia di litispendenza internazionale contenuta al tempo nella Convenzione di Bruxelles del 1968. Si rimanda in proposito a F. MARONGIU BuONAIUTI, Litispendenza e connessione internazionale, cit., p. 291 ss.; ID., Lis pendens and Related Actions in Civil and Commercial Matters Within the European Judicial Area, cit., p. 528 ss. 
decisione unica al fine di evitare risultati incompatibili ${ }^{35}$. Il regolamento sulla tutela dei dati personali appare accogliere, invece, nell'art. 81 un modello ibrido, basato sulla sola identità dell'oggetto delle due domande, senza un riferimento espresso all'ulteriore presupposto oggettivo della litispendenza europea costituito dall'identità di titolo, salvo quest'ultimo requisito sia da ritenersi implicito nella pur non chiarissima formulazione adottata dalla norma, che fa riferimento ad «azioni riguardanti lo stesso oggetto relativamente al trattamento [scil.: da parte] dello stesso titolare del trattamento o dello stesso responsabile del trattamento», ciò che potrebbe consentire di identificare nel trattamento dei dati, da cui le due o più domande pendenti traggono origine, il titolo la cui identità potrebbe ritenersi presupposta dalla norma ${ }^{36}$. L'incompleta identità delle domande al cui coordinamento la norma tende emerge anche sotto il profilo soggettivo, dal momento che essa richiede la sola identità del titolare ovvero del responsabile del trattamento, da identificarsi in linea di principio con la parte convenuta di ciascuna delle azioni concorrenti, stante la logica unilaterale che ispira le regole sulla giurisdizione contenute nel regolamento, le quali, come già rilevato, prendono in considerazione unicamente le azioni dei titolari dei dati nei confronti dei titolari o dei responsabili del trattamento, e non viceversa, e così neanche le azioni che possano eventualmente sorgere tra titolari e responsabili del trattamento nei loro reciproci rapporti ${ }^{37}$.

24. Peraltro, appare il caso di osservare che l'irrilevanza dell'identità del titolare dei dati al fine dell'applicazione del meccanismo di coordinamento tra procedimenti paralleli previsto dal regolamento sulla protezione dei dati personali potrebbe considerarsi andare di pari passo con il ruolo particolarmente ampio che il regolamento stesso, all'art. 80 , riserva in sede processuale ad organismi attivi nel settore della promozione della tutela dei dati personali, i quali potranno agire sia, ai sensi del par. 1 della norma ora evocata, per conto del titolare dei dati, sia, ai sensi del par. 2 della norma stessa, a tutela di un interesse diffuso alla regolarità del trattamento dei dati personali, indipendentemente da un mandato ricevuto dal titolare dei dati, a condizione che la legge dello Stato membro del foro lo preveda. Inoltre, appare doversi osservare che, nell'economia della materia considerata, è da ritenersi tendenzialmente più verosimile che un'esigenza di coordinamento possa sorgere tra procedimenti paralleli riguardanti violazioni delle disposizioni del regolamento in esame da parte di un determinato titolare o responsabile del trattamento, il quale, ove segua delle modalità di trattamento dei dati non compatibili con le disposizioni del regolamento, è suscettibile di reiterare le medesime violazioni nei confronti di una pluralità di titolari di dati personali, piuttosto che tra azioni proposte da un medesimo titolare dei dati nei riguardi di diversi titolari o responsabili del trattamento, posto che, ove azioni di quest'ultimo tipo si riferiscano al medesimo trattamento imputabile a più soggetti in veste di titolari ovvero di responsabili, il rimedio è, come già si è osservato, per un verso offerto a livello sostanziale dalle disposizioni stesse del regolamento ${ }^{38} \mathrm{e}$, per altro verso, da cercarsi ex ante e precisamente nel meccanismo del cumulo soggettivo contemplato dall'art. 8, par. 1, del regolamento "Bruxelles I-bis" 39.

35 Secondo la definizione attualmente contenuta nell'art. 30, par. 3, del regolamento n. 1215/2012 o "Bruxelles I-bis" e, in termini corrispondenti, nelle analoghe disposizioni contenute negli altri regolamenti che contemplano il medesimo meccanismo della connessione privativa.

${ }^{36}$ Può considerarsi deporre in questo senso il riferimento allo stesso titolo che compare nel considerando n. 144 del preambolo del regolamento, malgrado questo, evidenziando nuovamente una notevole imprecisione nella formulazione, lo ponga in alternativa rispetto al requisito dell'identità dell'oggetto, chiaramente posto dalla lettera dell'art. 81 del regolamento.

37 Secondo quanto si è già avuto modo di rilevare supra, II.A, la competenza giurisdizionale relativamente alle azioni proposte da questi altri soggetti deve ritenersi disciplinata dalle regole generali contenute nel regolamento n. 1215/2012 o "Bruxelles I-bis", secondo la ratio dell'art. 67 di quest'ultimo regolamento che risulta seguita anche dal preambolo del regolamento n. 2016/679, al considerando n. 147. Come si vedrà infra, par. 27, per la medesima ratio le regole in materia di litispendenza e connessione contenute nel regolamento Bruxelles I-bis troveranno applicazione nei rapporti tra azioni proposte da soggetti diversi dai titolari dei dati personali e tra queste e le azioni proposte dai titolari dei dati in base al regolamento in esame.

${ }^{38}$ Si deve ricordare, a questo proposito, che l'art. 82, par. 4, del regolamento n. 2016/679 prevede in ipotesi di questo tipo un regime di responsabilità solidale tra i diversi titolari ovvero responsabili del trattamento, per cui, per quanto attiene alle azioni di carattere risarcitorio, il titolare dei dati può dispensarsi dall'agire parallelamente nei confronti dei diversi soggetti, titolari ovvero responsabili del trattamento, cui la violazione sia imputabile, potendo invocare da uno solo di essi il risarcimento dell'intero danno patito. Naturalmente, questo meccanismo non è invocabile nel caso in cui il titolare dei dati richieda altro genere di provvedimenti, come la cancellazione o la rettifica dei dati oggetto del trattamento, dato che in questi casi dovrà agire nei confronti di ciascun titolare o responsabile del trattamento che detenga i dati in questione.

39 Si veda supra, II.A. Deve peraltro ricordarsi che anche ai fini della litispendenza nel sistema del regolamento "Bruxelles 
25. A ben vedere, il meccanismo di coordinamento tra procedimenti paralleli adottato dal regolamento sulla tutela dei dati personali presenta maggiore affinità con la disciplina della connessione privativa, propria del regolamento "Bruxelles I-bis" come già del precedente "Bruxelles I", piuttosto che con quella della litispendenza ${ }^{40}$. Infatti, come chiaramente risulta dal par. 3 della norma dell'art. 81 del regolamento in esame, essa, coerentemente con la già accennata piuttosto ampia identificazione dei requisiti per la sua applicazione, presuppone la solo parziale identità delle due domande. Ciò è reso evidente dal fatto che, a seguito della sospensione disposta dal giudice successivamente adito - la quale, analogamente a quanto avviene in caso di connessione ai sensi dell'art. 30 del regolamento "Bruxelles I-bis" e delle disposizioni corrispondenti degli altri regolamenti ispirati allo stesso modello, è meramente discrezionale - il giudice che ha sospeso il procedimento possa, a domanda di parte, declinare la propria giurisdizione unicamente quando entrambi i procedimenti siano pendenti in primo grado e il giudice adito per primo sia competente a conoscere delle due domande e la sua legge ne consenta la riunione in unico procedimento. Tali requisiti, come è noto, sono posti in termini corrispondenti ai fini di una declinatoria per connessione nella disciplina recata dal regolamento "Bruxelles I-bis", come già dal precedente regolamento "Bruxelles I", e trovano per l'appunto giustificazione nella non piena identità delle domande pendenti innanzi a giudici di Stati membri diversi ${ }^{41}$.

26. Per quanto, rispetto alla disciplina contenuta nel regolamento "Bruxelles I-bis" in materia, rispettivamente, di litispendenza e di connessione privativa, la disciplina del coordinamento tra procedimenti paralleli contenuta nel regolamento in materia di dati personali presenti evidenti caratteri di specialità che, conformemente alla regola generale di cui all'art. 67 del primo regolamento, comportano l'esclusione dell'applicazione della disciplina dettata da questo in tutti i casi in cui è applicabile quella contenuta in un regolamento avente ad oggetto una materia particolare, nondimeno deve ritenersi che un qualche spazio sussista, proprio nello spirito della regola di coordinamento ora evocata, per un'integra-

I-bis" il requisito dell'identità delle parti è stato interpretato dalla Corte di giustizia con una certa flessibilità, rivestendo decisiva rilevanza in proposito l'identità delle posizioni giuridiche soggettive fatte valere, malgrado la formale non coincidenza delle parti costituitesi in ciascun procedimento: si veda, con riferimento ad un caso di surrogazione in base ad un contratto di assicurazione, Corte di giustizia CE, sent. 19 maggio 1998, in causa C-351/96, Druot Assurances S.A. c. Consolidated Metallurgical Industries, in Raccolta, 1998, p. I-3075 ss. Si vedano in proposito F. PERSANO, Il rilievo della litispendenza internazionale nella Convenzione di Bruxelles del 1968: la nozione di «stesse parti», in Rivista di diritto internazionale privato e processuale, 1998, p. 713 ss.; F. Seatzu, The Meaning of «same parties» in Article 21 of the 1968 Brussels Jurisdiction and Judgments Convention, in European Law Review, 1999, p. 540 ss.; si rimanda anche a F. MARONGIU BUONAIUTI, Litispendenza e connessione internazionale, cit., p. 267, 269 ss.; ID., Lis pendens and Related Actions in Civil and Commercial Matters Within the European Judicial Area, cit., p. 543 ss. E' il caso di richiamare a questo proposito, inoltre, la soluzione contemplata dall'art. 19, par. 2, del regolamento n. 2201/2003 o "Bruxelles II-bis", nel quale, ai fini della litispendenza nei procedimenti relativi alla potestà genitoriale, per quanto riguarda l'identità soggettiva è fatto riferimento unicamente all'identità del minore della cui protezione si discute, indipendentemente dall'identità delle parti del procedimento, ciò che trova giustificazione sia, da un punto di vista sostanziale, nella centralità dell'interesse del minore nell'economia della materia considerata, sia, da un punto di vista procedurale, nella struttura dei procedimenti in materia, che possono sovente presentare carattere di volontaria giurisdizione. Si rimanda a quanto osservato in F. Marongiu Buonaiuti, Litispendenza e connessione internazionale, cit., p. 275 s.; ID., Lis pendens and Related Actions in Civil and Commercial Matters Within the European Judicial Area, cit., p. 545 s.

${ }^{40}$ Il considerando n. 144 del preambolo del regolamento n. 2016/679, del quale già si è rilevata, peraltro, la non del tutto felice formulazione, indica, infatti, i procedimenti paralleli oggetto del meccanismo di coordinamento in esame come connessi, richiamando per di più in proposito la definizione contenuta nell'art. 30 del regolamento Bruxelles I-bis, pur senza fare espresso riferimento a quest'ultima disposizione.

${ }^{41}$ Tali requisiti sono volti infatti ad assicurare, in presenza di azioni non identiche e quindi non intrinsecamente in grado di soddisfare ciascuna l'esigenza di tutela giurisdizionale di cui è espressione l'altra, che non si abbia per un verso a verificare il salto di un grado di giurisdizione, come potrebbe avvenire ove la causa innanzi al giudice successivamente adito penda in primo grado mentre l'altra innanzi al giudice precedentemente adito penda in appello o persino in ultima istanza innanzi a un giudice di legittimità, e per altro verso che non si verifichi un diniego di giustizia, ove il giudice precedentemente adito a favore del quale sia disposta la declinatoria non sia competente a conoscere della domanda proposta all'altro giudice. Il requisito per il quale la legge del giudice precedentemente adito deve consentire la riunione dei procedimenti è da intendersi volto per un verso a non vanificare il perseguimento dell'obiettivo della concentrazione dei procedimenti e per altro verso a rispettare l'autonomia procedurale dei singoli Stati membri, non imponendo ai giudici di dover far luogo a una riunione dei procedimenti in circostanze nelle quali le proprie norme di diritto processuale interno non lo consentirebbero. Ci si permette di rinviare in proposito a $\mathrm{F}$. Marongiu Buonaiuti, Litispendenza e connessione internazionale, cit., p. 469 ss.; ID., Lis pendens and Related Actions in Civil and Commercial Matters Within the European Judicial Area, cit., p. 527 s. 
zione della disciplina recata dal regolamento sulla protezione dei dati personali per quanto attiene agli aspetti che quest'ultima non regola espressamente e che trovano invece regolamentazione nella disciplina del coordinamento tra procedimenti paralleli contenuta nel regolamento "Bruxelles I-bis", con particolare riferimento alla determinazione del momento iniziale del procedimento, oggetto dell'art. 32 di quest'ultimo regolamento. La norma in questione reca a questo proposito una disciplina generalmente recepita in termini corrispondenti, salve alcune marginali precisazioni, in tutti gli altri regolamenti adottati nel settore della cooperazione giudiziaria in materia civile che recano regole ispirate al medesimo modello ${ }^{42}$.

27. Inoltre, sempre nella stessa ottica di sostanziale complementarità della disciplina in qualche misura generale recata dal regolamento "Bruxelles I-bis" rispetto alla disciplina speciale recata dal regolamento sulla protezione dei dati personali, deve ritenersi che la disciplina del coordinamento tra procedimenti paralleli recata dal primo regolamento, nelle due distinte ipotesi della litispendenza e della connessione privativa da essa contemplate, debba trovare applicazione nei casi che fuoriescono dall'ambito di applicazione delle disposizioni recate in proposito dal regolamento sulla protezione dei dati personali, posto che in tali casi l'applicazione dell'ordinaria disciplina della litispendenza e della connessione privativa recata dal regolamento "Bruxelles I-bis" non si rivelerebbe in grado di pregiudicare l'applicazione della disciplina speciale contenuta nell'art. 81 del regolamento sulla protezione dei dati personali. Quest'ultima, infatti, dovendo essere letta in stretto coordinamento con le regole di giurisdizione contenute nel medesimo capitolo del regolamento n. 2016/679, deve ritenersi applicabile unicamente alle situazioni di concorso tra più azioni introdotte sulla base di tali disposizioni ${ }^{43}$.

\section{Considerazioni conclusive: un'occasione mancata per un più efficace coordinamento tra atti adottati in settori diversi nell'ambito delle politiche materiali dell'Unione europea.}

28. L'esame che si è svolto delle disposizioni in materia di giurisdizione contenute nel regolamento n. 2016/679 in materia di protezione delle persone fisiche relativamente al trattamento dei dati

42 Tale disciplina, inserita inizialmente nell'art. 30 del regolamento n. 44/2001 o "Bruxelles I" allo scopo di ovviare quantomeno tendenzialmente ai problemi insiti nella diversità delle discipline processuali nazionali, evidenziati, nel silenzio della Convenzione di Bruxelles del 1968 sul punto, dalla Corte di giustizia CE, sent. 7 giugno 1984, in causa 129/83, Zelger c. Salinitri, in Raccolta, 1984, p. 2397 ss., contempla come rilevante al fine dell'adizione di un giudice il compimento del primo atto processuale rilevante, da identificarsi, a seconda delle modalità introduttive del giudizio previste dalla legge processuale del foro, col deposito dell'atto introduttivo presso l'ufficio del giudice, ovvero, ove la notifica al convenuto debba precedere il deposito, con la consegna dell'atto all'autorità competente per la notificazione. L'art. 32 del regolamento n. 1215/2012 o "Bruxelles I-bis" ha apportato alcune precisazioni, in realtà piuttosto tautologiche, alla regola, chiarendo che per autorità competente per la notificazione ai fini della regola debba intendersi la prima autorità che riceve l'atto da notificare - da identificarsi con la c.d. autorità mittente nel caso in cui la notificazione debba avere luogo in un paese diverso da quello del giudice adito - e disponendo che presso gli uffici giudiziari e le autorità competenti per la notificazione debba essere fatta annotazione delle date del deposito ovvero del ricevimento degli atti da notificare - ciò che è ragionevole attendersi avvenga già in base alle norme interne che di tali uffici e autorità disciplinano le funzioni - all'evidente fine di evitare incertezze nell'applicazione pratica della regola. Benché tali precisazioni non figurino nelle corrispondenti regole, ispirate al testo iniziale dell'art. 30 del regolamento $\mathrm{n}$. 44/2001, sulla determinazione del momento iniziale della pendenza del giudizio, contenute negli altri regolamenti che recano regole in materia di litispendenza e di connessione privativa, esse si presentano sufficientemente ovvie da considerarsi implicite anche in tale ulteriori disposizioni: si rinvia in proposito, con particolare riferimento alla regola contenuta nell'art. 14 del regolamento n. 650/2012 in materia di successioni, a F. Marongiu Buonaiuti, Article 14 - Seising of a Court, in A.-L. Calvo Caravaca, A. Davì, H.-P. Mansel (eds), The EU Succession Regulation. A Commentary, Cambridge, 2016, p. 234 ss., spec. p. 237 s.

43 Si rimanda in proposito alle considerazioni svolte supra, par. 22 e nota 29 , nel senso che, malgrado l'indicazione più restrittiva contenuta nel considerando n. 144 del preambolo del regolamento, del quale già si è rilevata la scarsa chiarezza espositiva, la disciplina del coordinamento tra procedimenti paralleli di cui all'art. 81 del regolamento n.2016/679 deve intendersi riferita non soltanto all'ipotesi in cui il procedimento innanzi al giudice successivamente adito presenti il carattere di un'azione, ovvero di un ricorso, avverso una decisione sfavorevole di un'autorità di controllo ai sensi dell'art. 78 del regolamento, bensì, data l'ampiezza della formulazione della norma e l'evidente eadem ratio, anche alle ipotesi in cui la previa pendenza di un procedimento parallelo che presenti i requisiti indicati nella norma sia rilevata da un giudice adito di un'azione rivolta contro un titolare o responsabile del trattamento ai sensi dell'art. 79. Si vedano invece, in aderenza all'interpretazione restrittiva qui criticata, P. DE Miguel Asensio, Aspectos internacionales del Reglamento general de protección de datos de la UE (I): cuestiones de competencia, cit., p. 4; P. FranzINA, Jurisdiction Regarding Claims for the Infringement of Privacy Rights, cit., p. 105 ss. 
personali evidenzia l'inadeguatezza del modo nel quale è stato affrontato il problema del coordinamento di tali disposizioni con la disciplina della competenza giurisdizionale in materia civile e commerciale attualmente recata dal regolamento n. 1215/2012 o "Bruxelles I-bis". Infatti, oltre alle perplessità di ordine generale che sono suscitate dalla prassi sempre più diffusa negli atti legislativi dell'Unione europea di inserire nei preamboli degli atti, ormai frequentemente estesi tanto quanto, se non più, del testo normativo dell'atto stesso, statuizioni che vanno al di là della mera esplicazione delle opzioni normative accolte nello strumento nel suo insieme o in sue singole disposizioni, configurandosi invece in molti casi nella sostanza come disposizioni integratrici del testo del regolamento ${ }^{44}$, la scelta di limitare tale coordinamento al mero inserimento di un succinto considerando nel preambolo si rivela insoddisfacente alla luce della pluralità e varietà dei profili problematici posti dalle interrelazioni tra le disposizioni dei due regolamenti. Infatti, come già notato, il considerando n. 147 del preambolo del regolamento $\mathrm{n}$. 2016/679 si limita nella sostanza a ripetere, senza nemmeno citarla espressamente, il contenuto della clausola di subordinazione, se così la si può chiamare mutuando la terminologia propria del diritto dei trattati, contenuta nell'art. 67 del regolamento "Bruxelles I-bis", per la quale, conformemente ad un criterio generale di specialità ratione materiae, le sue norme non pregiudicano l'applicazione delle regole di giurisdizione contenute in altri atti dell'Unione concernenti materie particolari ${ }^{45}$.

29. Chiaramente, se l'adozione di una formulazione così ampia può trovare giustificazione nell'atto che relativamente alla ripartizione della giurisdizione tra i giudici degli Stati membri in materia civile e commerciale presenta portata generale, in quanto la clausola in questione si presenta destinata ad applicarsi nei confronti della generalità degli atti, esistenti o futuri, che contengano regole di giurisdizione in materie che, rispetto all'ambito considerato, presentano carattere particolare, essa si rivela invece inadeguata all'interno di un atto come il regolamento n. 2016/679, le cui regole di giurisdizione presentano per un verso l'accennato carattere di specialità ratione materiae, senza per altro verso presentare il grado di completezza e sistematicità che è proprio della disciplina più generale contenuta nel regolamento "Bruxelles I-bis". In queste circostanze, sarebbe stato invece opportuno inserire, alternativamente, nel testo delle singole disposizioni contenenti, rispettivamente, $i$ criteri di giurisdizione ovvero la disciplina del coordinamento tra procedimenti paralleli, oppure in un'unica norma di chiusura collocata al termine del capitolo dedicato alla tutela giurisdizionale dei diritti riconosciuti dal regolamento n. 2016/679, specifiche disposizioni di coordinamento atte a chiarire in quali termini le regole in questione siano destinate ad essere integrate dalle disposizioni pertinenti del regolamento "Bruxelles I-bis". Inoltre, come già si è rilevato con riferimento alle regole concernenti il coordinamento tra procedimenti paralleli, la scelta di dettare in un atto che, nella parte pertinente, disciplina la giurisdizione in una materia particolare, regole largamente ispirate a quelle contenute nel regolamento "Bruxelles I-bis", ma che, pur sempre, se ne differenziano nei termini concreti in cui sono formulate, non appare, in assenza di evidenti ragioni di carattere sostanziale che giustifichino la differenziazione, foriero di chiarezza ed omogeneità della disciplina. Ben si potranno avere, infatti, come si è notato con specifico riferimento alle regole da ultimo evocate, situazioni ricadenti nella materia considerata nelle quali il coordinamento tra procedimenti paralleli verrà retto dalle disposizioni contenute nel regolamento concernente la materia in questione ed altre in cui troverà invece applicazione la disciplina generale contenuta nel regolamento "Bruxelles I-bis"46.

30. Infine, non può farsi a meno di rilevare che l'inadeguatezza del modo nel quale nel regolamento n. 2016/679 è stato curato il coordinamento delle regole da esso introdotte in materia di giurisdizione e di rapporti tra procedimenti paralleli con la disciplina contenuta in proposito nel regolamento "Bruxelles I-bis" si rivela, in ultima analisi, suscettibile di ostacolare, a causa del quadro di incertezza

${ }^{44}$ Si vedano, per alcuni rilievi critici in ordine all'ampio uso dei preamboli degli atti adottati in temi recenti nel settore della cooperazione giudiziaria in materia civile allo scopo di integrare il testo con ulteriori statuizioni di valenza più precettiva che meramente esplicativa, A. DAVİ, A. ZANoBETtI, Il nuovo diritto internazionale privato delle successioni nell'Unione europea, in Cuadernos de derecho transnacional, 2013, n. 2, p. 17, nota 55; ID., Il nuovo diritto internazionale privato europeo delle successioni, Torino, 2014, p. 23 s., nota 74.

${ }^{45} \mathrm{Si}$ veda in proposito supra, II.A.

46 Si rimanda a quanto osservato supra, III, spec. par. 27. 
normativa nel quale tali norme si troveranno ad essere applicate, il perseguimento dell'obiettivo di assicurare al titolare dei dati personali una tutela giurisdizionale effettiva dei diritti riconosciutigli dal regolamento stesso, che il legislatore dell'Unione ha per l'appunto inteso perseguire corredando la disciplina sostanziale contenuta nel regolamento di specifiche norme di giurisdizione per le azioni volte alla tutela dei diritti da esso garantiti ${ }^{47}$. In attesa che opportuni chiarimenti con riguardo ai diversi profili di incertezza che si sono rilevati giungano dalla giurisprudenza della Corte di giustizia, alla quale tali profili verranno prevedibilmente sottoposti, non può farsi a meno di manifestare l'impressione che l'introduzione nel regolamento sulla protezione delle persone fisiche relativamente al trattamento dei dati personali di un titolo apposito dedicato alla tutela giurisdizionale dei diritti abbia costituito un'occasione mancata per promuovere un maggiore e più accurato coordinamento dell'attività legislativa in settori differenti della sempre più vasta ed articolata sfera delle politiche materiali dell'Unione.

${ }^{47}$ Si veda supra, I, par. 2. 